\title{
ORDERING OF BINARY INTERSTITIAL SOLID SOLUTIONS
}

\author{
V. A. Volkov and S. I. Masharov
}

UDC 541.123.34:548.313.3.001

Ordering of binary interstitial solid solutions in a crystal with a BCC lattice is investigated. Two cases are considered: 1) interstitial atoms occupy interstices of only one type, 2) interstitial atoms occupy interstices of two types. It is demonstrated that in the second case, the order-disorder phase transformation is absent, and the solution remains ordered at all temperatures.

Keywords: interstitial solution, ordering, isotope solution.

A special place among interstitial solid solutions is occupied by multicomponent systems in which interstices are occupied by atoms of two and more species. In theoretical investigations of the interstitial atom distribution in the crystal, single-component solutions are conventionally studied (except isotope solutions [1]). However, the multicomponent systems possess a number of special features: ordering in them is described by more than one order parameter, and the energy of interaction of the interstitial atom with matrix atoms has several different values; moreover, the energy of interatomic bonding of interstitial atoms also has several different values. All this causes the special features in the temperature dependence of the interstitial atom distribution over the interstices. In the present work, these special features are investigated for binary interstitial solutions in a crystal-solvent with a BCC lattice neglecting the short-range order and taking into account the interaction of only the nearest neighbors. Two cases are considered: 1) interstitial atoms occupy only octa-interstices, and 2) interstitial atoms can occupy both octa- and tetrainterstices.

1. Let us consider a BCC crystal with $N_{1}$ and $N_{2}$ atoms of two different species incorporated into octainterstices. We subdivide all interstices into types 1 (in the centers of the boundaries) and 2 (in the middle of the edges). If $N_{1}+N_{2}<N$ ( $N$ is the number of interstices), the problem is reduced to finding the distribution of $N_{1}$ and $N_{2}$ atoms and $N_{v}$ vacancies $\left|N_{v}=N-N_{1}-N_{2}\right|$ over interstices of different types. Having introduced the probabilities of substitution of interstices of the $j$ th type by atoms of the $i$ th species and vacancies $p_{i}^{(j)}$ and $p_{v}^{(j)}$, we determine two order parameters:

$$
\eta=p_{1}^{(1)}-p_{2}^{(2)}, \quad \xi=p_{v}^{(1)}-p_{v}^{(2)}
$$

The probabilities $p_{1}^{(j)}, p_{2}^{(j)}$, and $p_{v}^{(j)}$ are expressed in terms of $\eta$ and $\xi$ as follows:

$$
\begin{array}{cc}
p_{1}^{(1)}=c_{1}+\frac{\eta}{2}, & p_{1}^{(2)}=c_{1}-\frac{\eta}{2}, \\
p_{2}^{(1)}=c_{2}+\frac{\eta+\xi}{2}, & p_{2}^{(2)}=c_{2}-\frac{\eta+\xi}{2}, \\
p_{v}^{(1)}=c_{v}+\frac{\xi}{2}, & p_{v}^{(2)}=c_{v}-\frac{\xi}{2} .
\end{array}
$$

Ural Federal University Named after the First President of Russia B. N. El'tsin, Ekaterinburg, Russia, e-mail: vavolk@mail.ru. Translated from Izvestiya Vysshikh Uchebnykh Zavedenii, Fizika, No. 9, pp. 88-92, September, 2012. Original article submitted May 11, 2010. 
The free energy of the solution is

$$
F=F_{0}+F_{\text {conf }}+F_{\mathrm{ph}},
$$

where $F_{0}$ is the free energy of the crystal-solvent, $F_{\text {conf }}$ is the configuration component of the free energy equal to

$$
\begin{gathered}
F_{\text {conf }}=u_{1} N_{1}+u_{2} N_{2}-2\left\{\left(N_{1}+N_{2}-N_{v}\right) u_{12}+2 N\left[\left(u_{12}-u_{11}\right)\left(c_{1}^{2}-\frac{\eta^{2}}{4}\right)\right.\right. \\
\left.+\left(u_{12}-u_{22}\right)\left(c_{2}^{2}-\frac{(\eta+\xi)^{2}}{4}\right)+u_{12}\left(c_{v}^{2}-\frac{\xi^{2}}{4}\right)\right\}+k T \frac{N}{2}\left\{\left(c_{1}+\frac{\eta}{2}\right) \ln \left(c_{1}+\frac{\eta}{2}\right)\right. \\
+\left(c_{2}+\frac{\eta+\xi}{2}\right) \ln \left(c_{2}+\frac{\eta+\xi}{2}\right)+\left(c_{v}+\frac{\xi}{2}\right) \ln \left(c_{v}+\frac{\xi}{2}\right)+\left(c_{1}-\frac{\eta}{2}\right) \ln \left(c_{1}-\frac{\eta}{2}\right) \\
\left.+\left(c_{2}-\frac{\eta+\xi}{2}\right) \ln \left(c_{2}-\frac{\eta+\xi}{2}\right)+\left(c_{v}-\frac{\xi}{2}\right) \ln \left(c_{v}-\frac{\xi}{2}\right)\right\},
\end{gathered}
$$

and $F_{\mathrm{ph}}$ is the phonon component of the free energy that for the model suggesting that atoms of different species in the interstices oscillate with the same frequencies $\omega_{1}$ and $\omega_{2}$ can be written as

$$
F_{\mathrm{ph}}=3 N_{1} k T \ln \left(2 \sinh \frac{\hbar \omega_{1}}{2 k T}\right)+3 N_{2} k T \ln \left(2 \sinh \frac{\hbar \omega_{2}}{2 k T}\right)
$$

In formulas (4) and (5), $u_{1}$ and $u_{2}$ are the interaction energies of the interstitial atoms of species 1 and 2 with the central atoms, and $u_{i k}$ is the energy of interaction of the nearest neighboring interstitial atoms of species $i$ and $k(i, k=$ 1,2) taken with the minus sign.

The expression for the free energy has been written disregarding the short-range order in the distribution of interstitial atoms; taking the correlation into account leads to the occurrence of the additional term in $F$ of the order of $\left(u_{i j} / k T\right)^{n}, n=1,2, \ldots$ [2]. The equilibrium $\eta$ and $\xi$ values at the given temperature are determined from the extremum conditions for $F$ :

$$
\frac{\partial F}{\partial \eta}=0, \quad \frac{\partial F}{\partial \xi}=0
$$

System of equations (6) has zero solution $\eta=\xi=0$ corresponding to the completely disordered state of the solution at all temperatures. Ordering is observed when the point $T_{0}\left(T_{0}>0\right)$ of absolute stability loss of this state arises. Expanding Eq. (6) in series in $\eta$ and $\xi$ in the vicinity of $T_{0}$, where $\eta<<1$ and $\xi<<1$, we obtain the following expression for $T_{0}$ :

$$
T_{0}=\frac{4}{k}\left\{w c_{1}\left(c^{*}-c_{1}\right)-\left[c_{1} u_{11}+\left(c^{*}-c_{1}\right) u_{22}\right]\left(1-c^{*}\right),\right.
$$

where $w=2 u_{12}-u_{11}-u_{22}$ is the ordering energy, $c_{1}+c_{2}=c^{*}$, and $c_{1}$ and $c_{2}$ are concentrations of the species: $c_{1}=N_{1} / N$ and $c_{2}=N_{2} / N$. Formula (7) is written when the expression for $F$ is chosen in the approximation disregarding the correlation. 
Let us consider some special cases:

a) $c^{*}=1$; all interstices are occupied by interstitial atoms. Then

$$
T_{0}=-\frac{4 w}{k} c_{1}\left(1-c_{1}\right)
$$

and ordering is possible when $w>0$.

b) $c_{2}=0$ and $c^{*}=c_{1}$; a single-component solid solution. In this case,

$$
T_{0}=-\frac{4}{k} u_{11} c_{1}\left(1-c_{1}\right)
$$

and ordering is observed when $u_{11}<0$. This result was obtained in [1].

c) $c_{2}=0$ but $c_{v} \neq 0$; analyzing the $T_{0}$ behavior as a function of $c_{1}$, we find that ordering for $w>0$ is possible when the composition satisfies the condition

$$
\left[\left(-\infty, c_{-}\right) \cap\left(0, c^{*}\right)\right] \cup\left[\left(0, c^{*}\right) \cap\left(c_{+}, \infty\right)\right] \neq \varnothing
$$

where $c_{-}$and $c_{+}$are $c_{1}$ values at which $T_{0}$ goes to zero; moreover, $c_{-}<c_{+}$and $w<0$ given that

$$
\left(c_{-}, c_{+}\right) \cap\left(0, c^{*}\right) \neq \varnothing
$$

d) $u_{11}=u_{22}=u_{12}=u$ (isotope solution); in this case,

$$
T_{0}=-u c^{*}\left(1-c^{*}\right)
$$

and ordering is observed when $u<0$. We note that consideration of the phonon contribution to $F$ does not influence at all the ordering pattern. This is due to the fact that frequencies of atoms of one species in different interstices are the same; for this reason, $F_{\mathrm{ph}}$ is independent of $\eta$ and $\xi$.

2. Now we consider systems in which ordering can proceed in octo- and tetra-interstices of the BCC lattices whose numbers are equal to $N$ and $2 N$, respectively. In this case, the concentrations of the interstitial components are

$$
c_{1}=\frac{N_{1}}{3 N}, \quad c_{2}=\frac{N_{2}}{3 N} .
$$

Two-component interstitial solutions with distribution of atoms over the interstices of two types were considered in [1] for the model of non-interacting interstitial atoms with completely occupied interstitial positions. Here we investigate a more general case when some interstices can remain vacant and consider the interaction of the interstitial atoms.

Let us define, as above, the order parameters by formula (1) and consider that octa-interstices are of the $1^{\text {st }}$ type and tetra-interstices are of the $2^{\text {nd }}$ type. For the probabilities of their occupation by atoms of different species, we have

$$
\begin{aligned}
& P_{1}^{0}=c_{1}+\frac{2}{3} \eta, P_{1}^{T}=c_{1}-\frac{1}{3} \eta, \\
& P_{v}^{0}=c_{v}+\frac{2}{3} \xi, P_{v}^{T}=c_{v}-\frac{1}{3} \xi,
\end{aligned}
$$




$$
P_{2}^{0}=c_{2}-\frac{2}{3}(\eta+\xi), P_{2}^{+}=c_{2}+\frac{1}{3}(\eta+\xi)
$$

Representing the free energy in the form given by Eq. (3), we obtain, considering that the atom of the $i$ th species oscillates with the same frequency in the initial interstice,

$$
\begin{gathered}
F_{\text {conf }}=c_{1} N\left(u_{1}^{0}+2 u_{T}^{0}\right)+c_{2} N\left(u_{1}^{0}+u_{2}^{T}\right)+\frac{2}{3} N\left(u_{1}^{0}-u_{1}^{T}\right) \eta \\
+\left(u_{2}^{T}-2 u_{2}^{0}(\eta+\xi)\right)-4 N\left\{c_{1}^{2} u_{11}+c_{2}^{2} u_{22}+2 c_{1} c_{2} u_{12}+\frac{1}{3}\left[\left(c_{1} u_{11}+c_{2} u_{22}\right) \eta\right.\right. \\
\left.\left.-\left(c_{2} u_{22}+c_{1} u_{11}\right)(\eta+\xi)\right]-\frac{2}{3}\left[u_{11} \eta^{2}+u_{22}(\eta+\xi)^{2}-2 u_{12} \eta(\eta+\xi)\right]\right\} \\
+k T N\left\{\left(c_{1}+\frac{2}{3} \eta\right) \ln \left(c_{1}+\frac{2}{3} \eta\right)-\left(c_{v}+\frac{2}{3} \xi\right) \ln \left(c_{v}+\frac{2}{3} \xi\right)+\left(c_{2}+\frac{1}{3}(\eta+\xi)\right)\right. \\
+\ln \left(c_{2}+\frac{1}{3}(\eta+\xi)\right)+\left(c_{1}-\frac{1}{3} \eta\right) \ln \left(c_{1}-\frac{1}{3} \eta\right)+\left(c_{v}-\frac{1}{3} \xi\right) \ln \left(c_{v}-\frac{1}{3} \xi\right) \\
F_{\text {ph }}=3 k T\left\{N_{1}^{0} \ln \left(2 \sinh \frac{\hbar \omega_{1}^{0}}{2 k T}\right)+N_{1}^{T} \ln \left(2 \sinh \frac{\hbar \omega_{1}^{T}}{2 k T}\right)+N_{2}^{0} \ln \left(2 \sinh \frac{\hbar \omega_{2}^{0}}{2 k T}\right)+N_{2}^{T} \ln \left(2 \sinh \frac{\hbar \omega_{2}^{T}}{2 k T}\right)\right\} .
\end{gathered}
$$

In these formulas, $u_{i}^{0}$ and $u_{i}^{T}$ are the interaction energies of the atom of the $i$ th species in octa- and tetra-interstice, respectively, with the nearest neighboring central atom, and $\omega_{i}^{0}$ and $\omega_{i}^{T}$ are the oscillation frequencies of the atom in these interstices. Considering that $N_{i}^{0}=N p_{i}^{0}$ and $N_{i}^{T}=2 N p_{i}^{T}$, we write down the conditions of extremum for the free energy with respect to $\eta$ and $\xi$ :

$$
\begin{gathered}
\ln \frac{\left(c_{1}+\frac{2}{3} \eta\right)\left(c_{2}+\frac{1}{3}(\eta+\xi)\right)}{\left(c_{1}-\frac{1}{3} \eta\right)\left(c_{2}-\frac{2}{3}(\eta+\xi)\right)}+\frac{\Delta U}{k T}-\frac{2}{k T}\left\{u_{11}\left(c_{1}-4 \eta\right)-u_{21}\left(c_{2}+\eta+\xi\right)\right. \\
+u_{12}\left(c_{2}-c_{1}+\frac{8}{3} \eta+\frac{4}{3} \xi\right)+3 f_{1}(T)=0, \\
\ln \frac{\left(c_{v}+\frac{2}{3} \xi\right)\left(c_{2}+\frac{1}{3}(\eta+\xi)\right)}{\left(c_{v}-\frac{1}{3} \xi\right)\left(c_{2}-\frac{2}{3}(\eta+\xi)\right)}+\frac{\Delta_{1} U}{k T}+\frac{2}{k T}\left\{u_{22}\left(c_{2}+\frac{4}{3}(\eta+\xi)\right)\right. \\
+u_{12}\left(c_{1}-\frac{4}{3} \eta\right)+3 f_{2}(T)=0 .
\end{gathered}
$$


Here

$$
\begin{gathered}
\Delta U=U_{1}^{0}-U_{1}^{T}+U_{2}^{T}-U_{2}^{0}, \quad \Delta_{1} U=U_{2}^{T}-U_{2}^{0}, \\
f_{1}(T)=\ln \left(\sinh \left(\frac{\hbar \omega_{1}^{0}}{k T}\right) \sinh \left(\frac{\hbar \omega_{2}^{T}}{2 k T}\right) / \sinh \left(\frac{\hbar \omega_{1}^{T}}{2 k T}\right) \sinh \left(\frac{\hbar \omega_{2}^{0}}{2 k T}\right)\right) \\
f_{2}(T)=\ln \left(\sinh \left(\frac{\hbar \omega_{2}^{T}}{2 k T}\right) / \sinh \left(\frac{\hbar \omega_{2}^{0}}{2 k T}\right)\right)
\end{gathered}
$$

Equations (17) generally represent a complex system of transcendental equations whose solution can be obtained only by numerical methods. However, one conclusion immediately follows at all temperatures, namely, it has no zero solution $\eta=\xi=0$, that is, the binary interstitial solution with two types of energetically non-equivalent interstices is always in the ordered state.

We now investigate some special cases of solutions of this system.

a) Single-component solution with vacancies $\left(c_{2}=0, c_{1}<1, c_{v}=1-c_{1}\right.$, and $\left.\xi=-\eta\right)$. The equation for $\eta$ has the form (neglecting the interaction of the interstitial atoms with each other)

$$
\ln \frac{\left(c_{1}+\frac{2}{3} \eta\right)\left(1-c_{1}+\frac{1}{3} \eta\right)}{\left(c_{1}-\frac{1}{3} \eta\right)\left(1-c_{1}-\frac{2}{3} \eta\right)}=\frac{-U_{1}^{0}-U_{1}^{T}}{k T} \eta+3 \ln \frac{\sinh \left(\frac{\hbar w_{1}^{0}}{2 k T}\right)}{\sinh \left(\frac{\hbar w_{1}^{T}}{k T}\right)}=0
$$

For $T \rightarrow \infty, \ln \left(\sinh \left(\frac{\hbar \omega_{1}^{0}}{2 k T}\right) / \sin \left(\frac{\hbar \omega_{1}^{T}}{2 k T}\right)\right) \rightarrow \ln \frac{\omega_{1}^{0}}{\omega_{2}^{T}}$; from this it follows that $\eta \rightarrow \eta_{0}$ at high temperatures, where $\eta_{0}$ is a solution of the equation

$$
\frac{\left(c_{1}+\frac{2}{3} \eta\right)\left(1-c_{1}+\frac{1}{3} \eta\right)}{\left(c_{1}-\frac{1}{3} \eta\right)\left(1-c_{1}-\frac{2}{3} \eta\right)}+\left(\frac{\omega_{1}^{0}}{\omega_{1}^{T}}\right)^{3}=10
$$

At low temperatures, $\ln \left(\sinh \left(\frac{\hbar \omega_{1}^{0}}{2 k T}\right) / \sinh \left(\frac{\hbar \omega_{1}^{T}}{2 k T}\right)\right)=\frac{\hbar\left(\omega_{1}^{0}-\omega_{1}^{T}\right)}{2 k T}$, and the equation for $\eta$ assumes the form

$$
\ln \frac{\left(c_{1}+\frac{2}{3} \eta\right)\left(1-c_{1}+\frac{1}{3} \eta\right)}{\left(c_{1}-\frac{1}{3} \eta\right)\left(1-c_{1}-\frac{2}{3} \eta\right)}=-\frac{\Delta_{1} U}{k T}-\frac{2}{3} \hbar \frac{\omega_{1}^{0}-\omega_{1}^{T}}{k T}
$$

b) A binary solution with completely occupied interstices $\left(c_{1}+c_{2}=1\right.$ and $\left.c_{v}=0\right)$. The equation for the equilibrium has the form 


$$
\begin{gathered}
\ln \frac{\left(c_{1}+\frac{2}{3} \eta\right)\left(c_{2}+\frac{1}{3} \eta\right)}{\left(c_{1}-\frac{1}{3} \eta\right)\left(c_{2}-\frac{2}{3} \eta\right)}+\frac{\Delta U}{k T}+\frac{2}{k T}\left\{c_{1}\left(u_{11}-u_{22}\right)+c_{2}\left(u_{12}-u_{22}\right)\right. \\
\left.+\frac{4}{3}\left(u_{11}+u_{22}-2 u_{12}\right) \eta\right\}+3 f_{1}(T)=0 .
\end{gathered}
$$

It can easily be seen that when $T \rightarrow \infty$, as in the previous case, $\eta$ tends to a constant value. At low temperatures, we have

$$
\begin{gathered}
\ln \frac{\left(c_{1}+\frac{2}{3} \eta\right)\left(c_{2}+\frac{1}{3} \eta\right)}{\left(c_{1}-\frac{1}{3} \eta\right)\left(c_{2}-\frac{2}{3} \eta\right)}+\frac{\Delta U}{k T}+\frac{2}{k T}\left\{c_{1}\left(u_{11}-u_{22}\right)+c_{2}\left(u_{12}-u_{22}\right)\right. \\
\left.+\frac{4}{3}\left(u_{11}+u_{22}-2 u_{12}\right) \eta\right\}+\frac{3}{2} \hbar\left(\omega_{1}^{0}+\omega_{2}^{T}-\omega_{1}^{T}-\omega_{2}^{0}\right)=0 .
\end{gathered}
$$

We note that in some solutions in which the frequencies $\omega_{i}^{0}$ and $\omega_{i}^{T}$ are sufficiently high, the region of low temperatures can be rather wide. Thus, for example, for cerium trihydride it extends up to room temperatures [1].

In an isotope alloy, $\Delta U=0$ and $u_{11}=u_{22}=u_{12}$, and Eq. (23) assumes the form of the equation obtained in [1, 3] for the model of non-interacting interstitial atoms; therefore, our result that considers this interaction is more general in character. The problem of ordering of the interstitial atoms in the FCC solvent can be considered analogously. The results will repeat the above-described ones.

\section{REFERENCES}

1. A. A. Smirnov, Theory of Interstitial Alloys [in Russian], Nauka, Moscow (1979).

2. S. I. Masharov and A. F. Rybalko, Fiz. Met. Metalloved., 47(4), 702 (1979).

3. V. A. Somenkov, I. R. Èntin, et al., Fiz. Tverd. Tela, 17, 2968 (1975). 